\title{
Extraction of Phthalic Acid from Aqueous Solution by Using Ionic Liquids: A Theoretical Study
}

\author{
Santhi Raju Pilli ${ }^{1}$, Kaustubha Mohanty ${ }^{2}$, Tamal Banerjee ${ }^{3}$ \\ Department of Chemical Engineering, Indian Institute of Technology Guwahati, \\ Guwahati - 781039, Assam, India \\ E-mail: ${ }^{1}$ ssanthirajup@gmail.com, ${ }^{2}$ kmohanty@iitg.ernet.in, ${ }^{3}$ tamalb@iitg.ernet.in
}

\begin{abstract}
Phthalic acid is an industrial chemical and it comes under the domain of endocrine disrupting chemicals (EDCs). Green solvents such as ionic liquids (ILs) possess good extractable capabilities for EDCs. COSMO-RS methodology is a widely accepted method for the design or selection of ionic liquids. COSMO-RS is a quantum chemical based method based on COSMO polarization charge densities. In this work the model has been used to screen the potential ionic liquids for the removal of phthalic acid from water. Five group of ILs with cations such as phosphonium, imidazolium, pyridinium, pyrolidinium and ammonium were screened at infinie dilution. A total of 34 cations and 29 anions i.e. 986 possible ILs were screened and their selectivities and capacities were predicted. The selectivities of the screened ILs are : phosphonium $>$ pyrolidinium $>$ imidazolium $>$ ammonim $>$ pyridinium. Some of the ionic liquids with selectivities were $[\mathrm{TBP}]\left[\mathrm{CF}_{3} \mathrm{SO}_{3}\right]$ (16057), $[\mathrm{OMPYR}]\left[\mathrm{BF}_{4}\right]$ (13265), $\left[\mathrm{C}_{4} \mathrm{DMIM}\right]\left[\mathrm{CF}_{3} \mathrm{SO}_{3}\right]$ (11678), [MNH][DEC] (9650) and $\left[\mathrm{C}_{8} \mathrm{MPY}\right]\left[\mathrm{CF}_{3} \mathrm{SO}_{3}\right]$ (4735). Experimental validation was done by comparing experimental logarithmic octanol-water partition coefficient values (Log $\left.K_{\text {ow }}\right)$ with COSMO-RS predicted data on phthalic acid. The results suggested that the ILs can be a possible solvent for the treatment of organic effluent rich water and wastewater.
\end{abstract}

Keywords: COSMO-RS; phthalic acid; endocrine disruption compounds; Gaussian 03; ionic liquids; wastewater treatment.

\section{Introduction}

In general phthalic acid esters (PAE) [1,2] co-disposed with domestic waste causes threat to the environment. Due to it, many phthalate esters have been termed as endocrine disruptors [1]. Disorders of the human body possibly involving EDCs in their pathological process includes vaginal adenocarcinoma, breast cancer, testicular cancer, generative problems, reduced fertility, reproductive tract abnormalities, sexual differentiation, skewed male/female sex ratios, hypospadias, loss of fetus, catamenial problems and premature pubarche [3,4]. Health effects due to endocrine disrupting compounds (EDCs) have been earlier studied in literature [5,6]. As per the biological study of physiology, an endocrine disrupting substance is a compound also known as environmental hormone, either natural or synthetic. United States Environmental Protection Agency (EPA) defined EDC as "an exogenous agent that interferes with synthesis, secretion, transport, metabolism, binding action, or elimination of natural blood-borne hormones that are present in the body and are responsible for homeostasis, reproduction, and developmental process" [4].

Wastewater treatment mainly depends upon adsorptive [7,8] and oxidative [9] processes. Various physicochemical methods have been reported in literature such as sedimentation, coagulation, ion exchange and filtration for the minimal levels of removal [10-12]. However these conventional physico-chemical remediation methods suffer from various disadvantages such as sludge generation, fouling due to ions or metals and large space. Consequently the membrane separation process is currently gaining a high scope $[8,13,14]$ for the removal of phthalic acid esters. Membrane based technologies offers potential applications in process industries and domestic requirements. Membrane separation processes have significant advantage due to low temperature of operation, low energy consumption, no phase change and simultaneous concentration and purification of products [15]. Limitations of permeable membranes is mainly due to their instability i.e. breakthrough of the immobilized phase in the pores which occur in most instances, except when a high breakthrough pressure through the membrane is maintained $[16,17]$.

In contrast to membrane separation processes, liquidliquid extraction using green solvents such as ionic liquids is becoming a better option for the treatment of such effluents. However the Ionic Liquids possessing of a cation and an anion can be infinite in number. For example, the cation can vary from imidazolium, phosphonium and pyridinium; while the anion can be chloride, tetrafluoroborate or hexafluorophosphate. Thus before experiments are conducted a proper screening needs to be conducted to choose the right combination of cation and anion. A quantum chemical based "COnductor-like Screening MOdel for Real Solvents" (COSMO-RS) method is used for the prediction of selectivity of compounds in aqueous medium at infinite dilution. The selectivity at infinite dilution is important since PAEs are present in trace amounts in water. This method a-priori predicts the activity coefficient of the solute i.e. phthalic 
acid in ionic liquid using the molecular structure as the only tool.

Several studies have been carried out by COSMO-RS methods to model the ionic liquids for the extraction of phenol [14,18], thiophene [19] and benzothiophene [20] Further it has been used for the desulfurization of diesel [21], extraction of cresols [22] and aromatic compounds [23]. Recently the model was applied for the removal of pesticides such as PCP and DDT [24], ethylene glycol [25] and extraction of dibenzothiophene, diphenyl sulfide, diphenyldisulfide, tetralin and benzene [26].

However the extraction of phthalic acid from aqueous phase using ionic liquids via COSMO-RS method is not reported till date. In this work an attempt have been done theoretically to extract the phthalic acid from aqueous phase using ionic liquid by COSMO-RS method. Combinations of 34 cations with 29 anions i.e. total of 986 possible ILs were used to determine the best extractant for

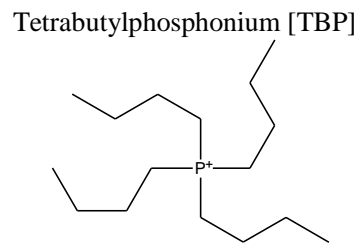

1-Hexyl-1-methyl-pyrrolidinium [HMPYR $]^{+}$

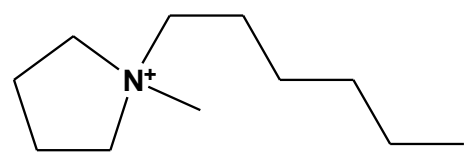

1-Octyl-3-methylimidazolium $\left[\mathrm{C}_{8} \mathrm{MIM}\right]^{+}$

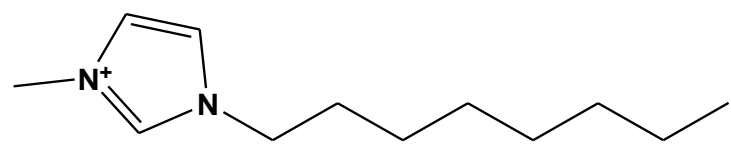

1-Hexyl-4-methylpyridinium $\left[\mathrm{C}_{6} \mathrm{MPY}\right]^{+}$

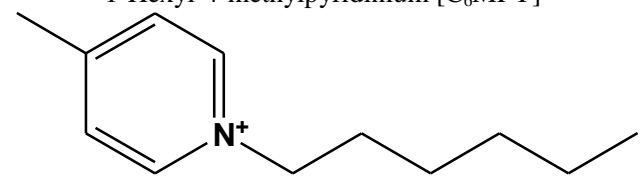

the removal of EDCs from aqueous solution. Figure 1 and 2 shows the structures of some of higher carbon chain length cations and structures of some of anions used in this study.

\section{Computational Details}

COSMO (Conductor like Screening MOdel) was first introduced by Klamt and Schüürmann [27] in 1993. The statistical thermodynamics extension termed as COSMORS. 'Conductor-like Screening Model for Real Solvents'. In this method the molecules are considered as collection of surface segments and chemical potential of each surface segment is self-consistently found from statistical mechanical calculation. The segment activity coefficient obtained from the difference in activity coefficient of segment between the pure liquid and mixtures. The summation of surface activity coefficient gives the activity coefficient of molecule. The details and the calculation procedure are given by [28] and [29].

Octyldiethylmethylphosphonium [ODEMP $]^{+}$

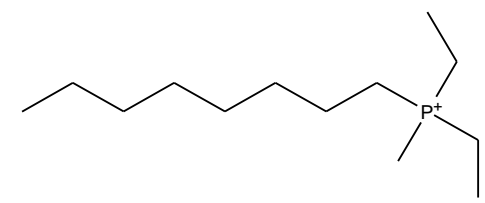

1-Octyl-1-methyl- pyrrolidinium [OMPYR]

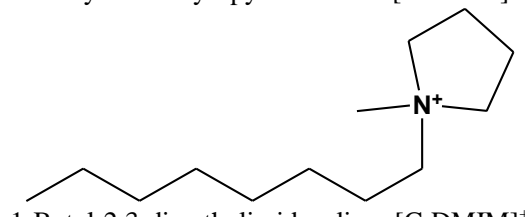

1-Butyl-2,3-dimethylimidazolium $\left[\mathrm{C}_{4} \mathrm{DMIM}\right]^{+}$

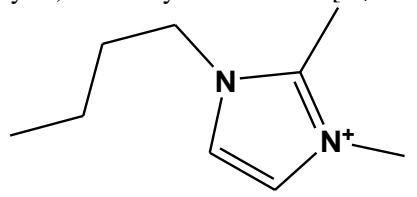

1-Octyl-4-methylpyridinium $\left[\mathrm{C}_{8} \mathrm{MPY}\right]^{+}$

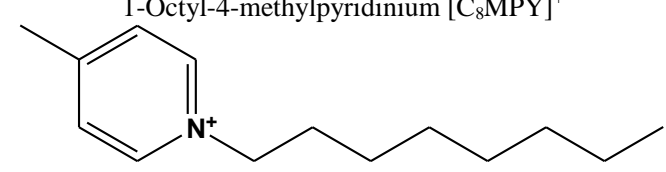

Figure 1. Examples of higher carbon chain length cations used in this study.

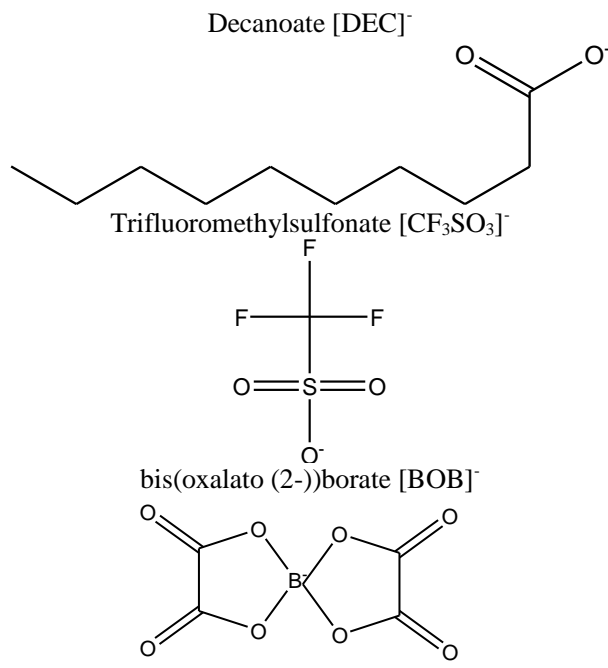

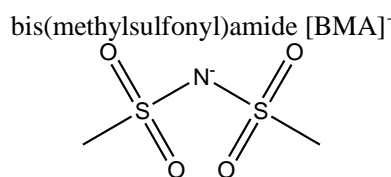

Tetrafluoroborate $\left[\mathrm{BF}_{4}\right]$<smiles>F[B-](F)(F)F</smiles>

Hexafluoro phosphate $\left[\mathrm{PF}_{6}\right]$

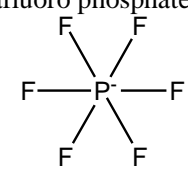

Figure 2. Examples of anions used in this study. 
The interaction energies within the segments and the methodological analysis for obtaining the equations are reported in our earlier work $[30,31]$. We have reimplemented the COSMO-SAC model as given by Sandler and Lin [28]. The COSMO file containing the ideal screening charges were obtained from the GAUSSIAN03 [32] package using the MOLDEN visualization freeware [24,33]. The interaction energies are described by the COSMO-RS parameters. Parameter values used in the COSMO-RS model is surface area of a standard segment $\left(a_{\text {eff }}\right)=6.25 \AA^{2}$, misfit constant for misfit energy interaction $\left(\alpha^{\prime}\right)=8896\left(\mathrm{kcal} \AA^{4}\right) /\left(\mathrm{mol} \mathrm{\textrm {e } ^ { 2 }}\right)$, constant for hydrogenbonding interaction $\left(c_{h b}\right)=54874\left(\mathrm{kcal} \AA^{4}\right) /\left(\mathrm{mol} \mathrm{e} \mathrm{e}^{2}\right)$, and the cut off value for hydrogen-bonding interactions $\left(\sigma_{h b}\right)=0.0085 \mathrm{e} / \AA^{2}$ and are taken from previous work $[30,31]$.

\section{Results and Discussion}

For the screening of the Ionic Liquids we define two parameters namely selectivity and capacity at infinite dilution. Selectivity (S) is defined as the ratio of the water species in ionic liquid rich phase (extract) to the composition of phthalic acid in ionic liquid rich phase [24]. The mathematical expression can be written as:

$S_{12, \max }=S_{12}^{\infty}=\left(\frac{\gamma_{2}^{\infty}}{\gamma_{1}^{\infty}}\right)^{I L \text { phase }}\left(\frac{\gamma_{1}^{\infty}}{\gamma_{2}^{\infty}}\right)^{\text {Water phase }} \approx\left(\frac{\gamma_{2}^{\infty}}{\gamma_{1}^{\infty}}\right)^{I L \text { phase }}$

where IL represents ionic liquid, $\gamma_{1}^{\infty}$ is the activity coefficient of phthalic acid species at infinite dilution and $\gamma_{2}^{\infty}$ activity coefficient of water component at infinite dilution. The activity coefficients at infinite dilution $\left(\gamma^{\infty}\right)$ are very vital to judge a chemical solvent. The aim of the infinite dilution is most cumbersome job as it refers to the removal of last trace of the component from aqueous phase. The prediction of activity coefficients at infinite dilution was carried out because solvent effects were found mostly when the concentration of solute i.e. phthalic acid is infinitely small. Nevertheless, infinite dilution also employs to the position where the presence of a trace amount of phthalic acid poses the main trouble.

The solvent capacity is directly used to measure the quantity of ionic liquid required for the extraction of phthalic acid species. It means that the capability to dissolve maximum amount of phthalic acid species present in the solvent. The capacity (C) can be expressed in terms of IDAC:

$C_{12}^{\infty}=\left(\frac{1}{\gamma_{1}^{\infty}}\right)^{I L \text { Phase }}$

The subscripts ' 1 ' indicates phthalic acid species in ionic liquid phase and the subscripts ' 2 ' indicates ionic liquid. The selectivity and capacity strongly depends on solventsolute interaction effect. The extraction capacity depends on anion volume and interaction energy between cation and anion. In this work all the activity coefficients were calculated at infinite dilution. As the values of the activity coefficients are less than unity, the interaction between the solute and the ionic liquid gets stronger. A screening study was done for 986 possible ionic liquids (a combination of 34 cations combined with 29 anions) and to determine the best extractant. Table 1 and 2 shows the list of anions and cations studied in this work with its chemical structure, chemical formulae and molecular weight. However the selections of ionic liquids are not only based on the selectivity and capacity, but also depend on properties such as hydrophobicity and toxicity. In case the best selective ionic liquid is toxic in nature, the second best ionic liquid will be chosen provided it considers the hydrophobicity and hazardous activity of solvent. Previous studies reported [33] that ionic liquids having affinity to water are undesirable for liquid-liquid extraction.

\subsection{Experimental Validation}

Before proceeding with predictions, COSMO-RS model was first benchmarked with reported (Table 3) octanolwater partition coefficient values $\left(K_{\text {ow }}\right)$. The equilibrium distribution of an organic chemical (phthalic acid in this case) between water and octanol can be expressed through octanol-water partition coefficient data. For predicting the effect of a chemical to partition to water, $K_{\text {ow }}$ is an important physical constant.

Primary benchmarking studies have been carried out for phthalic acid by comparing present predictions with the experimental values $\left(K_{\mathrm{ow}}\right)_{\exp }$ [34]. Predicted partition (Log $\left.\left(K_{\text {ow }}\right)_{\text {pred }}\right)$ coefficients were calculated by the following equation.

$$
K_{O W, i}=\frac{C_{i}^{O R}}{C_{i}^{W R}}=\frac{C_{t o t}^{O R} x_{i}^{O R}}{C_{t o t}^{W R} x_{i}^{W R}}=\frac{C_{t o t}^{O R} \gamma_{i, W R}^{\infty}}{C_{t o t}^{W R} \gamma_{i, O R}^{\infty}}=0.148 \frac{\gamma_{i, W R}^{\infty}}{\gamma_{i, O R}^{\infty}}
$$

Results from Table 3 show that the Log $\left(K_{\text {ow }}\right)_{\text {pred }}$ and $\log \left(K_{\text {ow }}\right)_{\exp }$ values of phthalic acid are close to each other i.e. 0.639 and 0.722 respectively (Table 3 ). Thus it serves as a validation basis for our comparison. Additionally, previous studies from our group have reported the successful validation of IDAC values in ILs [14,24]. Some of the applications are desulphurization [19], denitrification [35], phenol removal [14] and recently PCP and DDT removal [24]. Keeping this in mind, we will now proceed with the screening of various cations. Selectivity values of favorable ionic liquids and their corresponding aqueous phase activity coefficient of phthalic acid are shown in Tables 4-6.

\subsection{Selectivity of Phosphonium Based Ionic Liquids}

Selectivity values are calculated from eq. (1). Fig. 3 describes the selectivity values of phosphonium based ionic liquids for phthalic acid. In the screening process, [TBP] $\left[\mathrm{CF}_{3} \mathrm{SO}_{3}\right]$, [ODEMP] $\left[\mathrm{CF}_{3} \mathrm{SO}_{3}\right]$, [HDEMP] $-\left[\mathrm{CF}_{3} \mathrm{SO}_{3}\right]$ and [TIBMP] $\left[\mathrm{CF}_{3} \mathrm{SO}_{3}\right]$ gave the high selectivity of 16057 , 13655,11783 and 11670 respectively for the extraction of phthalic acid. High values were obtained by phosphonium based ionic liquid [TBP] $\left[\mathrm{CF}_{3} \mathrm{SO}_{3}\right]$ (16057) which is the highest among all the cation groups studied i.e. imidazolium, pyridinium, pyrrolidinium and ammonium. The high selectivity is due to the longer carbon chain length of $[\mathrm{TBP}]\left[\mathrm{CF}_{3} \mathrm{SO}_{3}\right]$. The higher vdW area helps in accommodating more number of phthalic acid molecules. The anion $\left[\mathrm{CF}_{3} \mathrm{SO}_{3}\right]^{-}$, a trifluoromethanesulfonic acid or triflic acid posses high thermal and chemical stability. It is known for its hydrophobicity, thereby restricting the entry of water molecules. The selectivity increases with an increase in the carbon chain length on phosphonium cation. 
Table 1. List of anions studied in this work.

\begin{tabular}{|c|c|c|c|}
\hline S. No. & Name of Anion & Acronym & $\begin{array}{c}\text { Chemical } \\
\text { Formulae/ } \\
\text { Mol. Wt. }\left(\mathrm{g} / \mathrm{cm}^{3}\right)\end{array}$ \\
\hline 1 & Acetate & {$[\mathrm{OAc}]^{-}$} & $\mathrm{C}_{2} \mathrm{H}_{3} \mathrm{O}_{2} / 59.01$ \\
\hline 2 & Ttetracyanoborate & {$\left[\mathrm{B}(\mathrm{CN})_{4}\right]^{-}$} & $\mathrm{C}_{4} \mathrm{BN}_{4} / 114.88$ \\
\hline 3 & Tetrafluoroborate & {$\left[\mathrm{BF}_{4}\right]^{-}$} & $\mathrm{BF}_{4}{ }^{-} / 86.80$ \\
\hline 4 & Bis(methylsulfonyl)amide & {$[\mathrm{BMA}]^{-}$} & $\begin{array}{l}\mathrm{C}_{2} \mathrm{H}_{6} \mathrm{NO}_{4} \mathrm{~S}_{2} / 172.2 \\
0\end{array}$ \\
\hline 5 & Bis(oxalato (2-))borate & {$[\mathrm{BOB}]^{-}$} & $\mathrm{C}_{8} \mathrm{H}_{14} \mathrm{BO}_{4} / 249.00$ \\
\hline 6 & $\begin{array}{l}\text { Bis(trifluoromethylsulfon } \\
\text { yl)amide }\end{array}$ & {$[\mathrm{BTA}]^{-}$} & $\begin{array}{l}\mathrm{C}_{2} \mathrm{~F}_{6} \mathrm{NO}_{4} \mathrm{~S}_{2} / 280.1 \\
4\end{array}$ \\
\hline 7 & Trifluoro-methylsulfonate & {$\left[\mathrm{CF}_{3} \mathrm{SO}_{3}\right]^{-}$} & $\mathrm{CF}_{3} \mathrm{O}_{3} \mathrm{~S} / 148.95$ \\
\hline 8 & Methylsulfonate & {$\left[\mathrm{CH}_{3} \mathrm{SO}_{3}\right]^{-}$} & $\mathrm{CH}_{3} \mathrm{O}_{3} \mathrm{~S} / 95.09$ \\
\hline 9 & Methyl sulfate & {$\left[\mathrm{CH}_{3} \mathrm{SO}_{4}\right]^{-}$} & $\mathrm{CH}_{4} \mathrm{O}_{4} \mathrm{~S} / 112.10$ \\
\hline 10 & Ethylsulfate & {$\left[\mathrm{C}_{2} \mathrm{H}_{5} \mathrm{SO}_{4}\right]^{-}$} & $\mathrm{C}_{2} \mathrm{H}_{6} \mathrm{O}_{4} \mathrm{~S} / 126.13$ \\
\hline 11 & Octylsulfate & {$\left[\mathrm{C}_{8} \mathrm{H}_{17} \mathrm{SO}_{4}\right]^{-}$} & $\mathrm{C}_{8} \mathrm{H}_{18} \mathrm{O}_{4} \mathrm{~S} / 210.29$ \\
\hline 12 & Chloride & {$[\mathrm{Cl}]^{-}$} & $\mathrm{Cl}^{-} / 35.45$ \\
\hline 13 & Dimethylphosphate & {$\left[\mathrm{DMPO}_{4}\right]^{-}$} & $\begin{array}{l}\mathrm{C}_{2} \mathrm{H}_{7} \mathrm{O}_{4} \mathrm{P} / 12126.0 \\
4\end{array}$ \\
\hline 14 & Hydrogensulfate & {$\left[\mathrm{HSO}_{4}\right]^{-}$} & $\mathrm{H}_{2} \mathrm{O}_{4} \mathrm{~S} / 98.07$ \\
\hline 15 & $\begin{array}{l}\mathrm{N}- \\
\text { methylsulfonylacetamide }\end{array}$ & {$[\mathrm{MAcA}]^{-}$} & $\begin{array}{l}\mathrm{C}_{3} \mathrm{H}_{7} \mathrm{NO}_{3} \mathrm{~S} / 137.1 \\
5\end{array}$ \\
\hline 16 & $\begin{array}{l}2-(2- \\
\text { methoxyethoxy)ethylsulfa } \\
\text { te }\end{array}$ & $\begin{array}{l}\text { [MDEGSO } \\
\left.{ }_{4}\right]^{-}\end{array}$ & $\mathrm{C}_{5} \mathrm{H}_{12} \mathrm{O}_{6} \mathrm{~S} / 200.21$ \\
\hline 17 & Dicyanamide & {$\left[\mathrm{N}(\mathrm{CN})_{2}\right]^{-}$} & $\mathrm{C}_{2} \mathrm{~N}_{3} / 60.8$ \\
\hline 18 & Hexafluorophosphate & {$\left[\mathrm{PF}_{6}\right]^{-}$} & $\mathrm{F}_{6} \mathrm{P} / 144.96$ \\
\hline 19 & Thiocyanate & {$[\mathrm{SCN}]^{-}$} & CNS/58.08 \\
\hline 20 & p-toluenesulfonate & {$[\mathrm{TOS}]^{-}$} & $\mathrm{C}_{7} \mathrm{H}_{7} \mathrm{O}_{3} \mathrm{~S} / 171.19$ \\
\hline 21 & Salicylate & {$[\mathrm{Sal}]^{-}$} & $\mathrm{C}_{7} \mathrm{H}_{5} \mathrm{O}_{3} / 137.11$ \\
\hline 22 & Nitrate & {$\left[\mathrm{NO}_{3}\right]^{-}$} & $\mathrm{NO}_{3} / 62.01$ \\
\hline 23 & Trifluoroacetate & {$\left[\mathrm{CF}_{3} \mathrm{COO}\right]^{-}$} & $\mathrm{C}_{2} \mathrm{~F}_{3} \mathrm{O}_{2} / 113.01$ \\
\hline 24 & Diethyl phosphate & {$[\mathrm{DEP}]^{-}$} & $\mathrm{C}_{4} \mathrm{H}_{11} \mathrm{O}_{4} \mathrm{P} / 154.10$ \\
\hline 25 & Decanoate & {$[\mathrm{DEC}]^{-}$} & $\mathrm{C}_{10} \mathrm{H}_{19} \mathrm{O}_{2} / 171.25$ \\
\hline 26 & Dibutyl phosphate & {$[\mathrm{DBP}]^{-}$} & $\begin{array}{l}\mathrm{C}_{18} \mathrm{H}_{19} \mathrm{O}_{4} \mathrm{P} / 210.20 \\
8\end{array}$ \\
\hline 27 & Bromide & {$[\mathrm{Br}]^{-}$} & $\mathrm{Br}^{-} / 79.90$ \\
\hline 28 & Dihydrogen phosphate & {$\left[\mathrm{DHPO}_{4}\right]^{-}$} & $\mathrm{H}_{3} \mathrm{O}_{4} \mathrm{P} / 97.99$ \\
\hline 29 & Perchlorate & {$\left[\mathrm{ClO}_{4}\right]^{-}$} & $\mathrm{ClO}_{4} / 99.45$ \\
\hline
\end{tabular}

Table 3. Physical and chemical properties of phthalic acid.

\begin{tabular}{ll}
\hline Chemical Formula & $\mathrm{C}_{8} \mathrm{H}_{6} \mathrm{O}_{4}$ \\
\hline CAS No.: & $88-99-3$ \\
Molecular Mass: & $166.14 \mathrm{~g} / \mathrm{mol}$ \\
Solubility in water: poor & $0.574 \mathrm{~g} / 100 \mathrm{ml}$ \\
& at $\left.20^{\circ} \mathrm{C}\right)$ \\
Density: & $1.593 \mathrm{~g} / \mathrm{cm}^{3}$ \\
Dissociation constant $\left(\mathrm{p} K_{\mathrm{a}}\right):$ & 2.98 \\
Octanol/water partition coefficient & 0.722 \\
$\left(\right.$ Log $\left.K_{o-w}\right)$ Exp & \\
Octanol/water partition coefficient & 0.641 \\
$\left(\right.$ Log $\left.K_{o-w}\right)$ Pred & \\
\hline
\end{tabular}

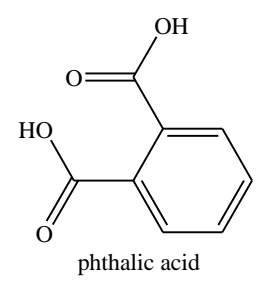

We also observe that the anion $\left[\mathrm{CF}_{3} \mathrm{SO}_{3}\right]^{-}$is common for many high selective cations of phosphonium group as compared to the common anions such as $\left[\mathrm{BF}_{4}\right]^{-}$and $[\mathrm{BMA}]$. From Table 4 and Fig. 3, cation $[\mathrm{TBP}]^{+}$with anion $\left[\mathrm{BCN}_{4}\right]^{-}$ the predicted selectivity is 496 though its corresponding aqueous phase activity coefficient is 18.483 . This shows that the $[\mathrm{TIBMP}]\left[\mathrm{BCN}_{4}\right]^{-}$is highly insoluble in water.
Table 2. List of cations studied in this work.

\begin{tabular}{|c|c|c|c|}
\hline S. No. & Name of Anion & Acronym & $\begin{array}{c}\text { Chemical } \\
\text { Formulae/ } \\
\text { Mol. Wt. }\left(\mathrm{g} / \mathrm{cm}^{3}\right)\end{array}$ \\
\hline 1 & $\begin{array}{l}\text { 1-ethtyl-3- } \\
\text { methylimidazolium }\end{array}$ & {$\left[\mathrm{C}_{2} \mathrm{MIM}\right]^{+}$} & $\mathrm{C}_{6} \mathrm{H}_{11} \mathrm{~N}_{2} / 111.164$ \\
\hline 2 & $\begin{array}{l}\text { 1-butyl-3- } \\
\text { methylimidazolium }\end{array}$ & {$\left[\mathrm{C}_{4} \mathrm{MIM}\right]^{+}$} & $\mathrm{C}_{8} \mathrm{H}_{17} \mathrm{~N}_{2} / 139.218$ \\
\hline 3 & $\begin{array}{l}\text { 1-methyl-3- } \\
\text { pentylimidazolium }\end{array}$ & {$\left[\mathrm{C}_{5} \mathrm{MIM}\right]^{+}$} & $\mathrm{C}_{9} \mathrm{H}_{17} \mathrm{~N}_{2} / 153.244$ \\
\hline 4 & $\begin{array}{l}\text { 1-hexyl-3- } \\
\text { methylimidazolium }\end{array}$ & {$\left[\mathrm{C}_{6} \mathrm{MIM}\right]^{+}$} & $\mathrm{C}_{10} \mathrm{H}_{19} \mathrm{~N}_{2} / 167.271$ \\
\hline 5 & $\begin{array}{l}\text { 1-octyl-3- } \\
\text { methylimidazolium }\end{array}$ & {$\left[\mathrm{C}_{8} \mathrm{MIM}\right]^{+}$} & $\mathrm{C}_{12} \mathrm{H}_{23} \mathrm{~N}_{2} / 195.186$ \\
\hline 6 & 1,3-dimethylimidazolium & {$\left[\mathrm{C}_{1} \mathrm{MIM}\right]^{+}$} & $\mathrm{C}_{5} \mathrm{H}_{9} \mathrm{~N}_{2} / 97.138$ \\
\hline 7 & $\begin{array}{l}\text { Methyl-methyl-methyl- } \\
\text { imidazolium }\end{array}$ & {$\left[\mathrm{C}_{1} \mathrm{DMIM}\right]^{+}$} & $\mathrm{C}_{6} \mathrm{H}_{11} \mathrm{~N}_{2} / 111.164$ \\
\hline 8 & $\begin{array}{l}\text { 1-ethyl-2,3- } \\
\text { dimethylimidazolium }\end{array}$ & {$\left[\mathrm{C}_{2} \mathrm{DMIM}\right]^{+}$} & $\mathrm{C}_{7} \mathrm{H}_{13} \mathrm{~N}_{2} / 125.191$ \\
\hline 9 & $\begin{array}{l}\text { 1-butyl-2,3- } \\
\text { dimethylimidazolium }\end{array}$ & {$\left[\mathrm{C}_{4} \mathrm{DMIM}\right]^{+}$} & $\mathrm{C}_{9} \mathrm{H}_{17} \mathrm{~N}_{2} / 153.244$ \\
\hline 10 & $\begin{array}{l}\text { 1-ethyl-4- } \\
\text { methylpyridinium }\end{array}$ & {$\left[\mathrm{C}_{2} \mathrm{MPY}\right]^{+}$} & $\mathrm{C}_{8} \mathrm{H}_{12} \mathrm{~N}_{2} / 122.187$ \\
\hline 11 & $\begin{array}{l}\text { 1-butyl-4- } \\
\text { methylpyridinium }\end{array}$ & {$\left[\mathrm{C}_{4} \mathrm{MPY}\right]^{+}$} & $\mathrm{C}_{10} \mathrm{H}_{16} \mathrm{~N} / 150.24$ \\
\hline 12 & $\begin{array}{l}\text { 1-hexyl-4- } \\
\text { methylpyridinium }\end{array}$ & {$\left[\mathrm{C}_{6} \mathrm{MPY}\right]^{+}$} & $\mathrm{C}_{12} \mathrm{H}_{20} \mathrm{~N} / 178.293$ \\
\hline 13 & $\begin{array}{l}\text { 1-octyl-4- } \\
\text { methylpyridinium }\end{array}$ & {$\left[\mathrm{C}_{8} \mathrm{MPY}\right]^{+}$} & $\mathrm{C}_{14} \mathrm{H}_{24} \mathrm{~N} / 206.347$ \\
\hline 14 & $\begin{array}{l}\text { 1,1-dimethyl- } \\
\text { pyrrolidinium }\end{array}$ & 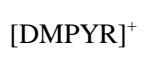 & $\mathrm{C}_{6} \mathrm{H}_{14} \mathrm{~N} / 100.182$ \\
\hline 15 & $\begin{array}{l}\text { 1-1-dipropyl- } \\
\text { pyrrolidinium }\end{array}$ & {$\left[_{\mathrm{DPPYR}}\right]^{+}$} & $\mathrm{C}_{10} \mathrm{H}_{22} \mathrm{~N} / 156.288$ \\
\hline 16 & $\begin{array}{l}\text { 1-butyl-1-ethyl- } \\
\text { pyrrolidinium }\end{array}$ & {$[\mathrm{BEPYR}]^{+}$} & $\mathrm{C}_{10} \mathrm{H}_{22} \mathrm{~N} / 156.288$ \\
\hline 17 & $\begin{array}{l}\text { 1-butyl-1-methyl- } \\
\text { pyrrolidinium }\end{array}$ & {$[\mathrm{BMPYR}]^{+}$} & $\mathrm{C}_{9} \mathrm{H}_{20} \mathrm{~N} / 142.261$ \\
\hline 18 & $\begin{array}{l}\text { 1-hexyl-1-methyl- } \\
\text { pyrrolidinium }\end{array}$ & {$[\mathrm{HMPYR}]^{+}$} & $\mathrm{C}_{11} \mathrm{H}_{24} \mathrm{~N} / 170.314$ \\
\hline 19 & $\begin{array}{l}\text { 1-octyl-1-methyl- } \\
\text { pyrrolidinium }\end{array}$ & {$[\mathrm{OMPYR}]^{+}$} & $\mathrm{C}_{13} \mathrm{H}_{28} \mathrm{~N} / 198.368$ \\
\hline 20 & $\begin{array}{l}\text { Benzyltrimethyl- } \\
\text { ammonium }\end{array}$ & {$[\mathrm{BETNH}]^{+}$} & $\mathrm{C}_{10} \mathrm{H}_{10} \mathrm{~N} / 150.24$ \\
\hline 21 & $\begin{array}{l}\text { Butyltrimethyl- } \\
\text { ammonium }\end{array}$ & {$[\mathrm{BTNH}]^{+}$} & $\mathrm{C}_{7} \mathrm{H}_{18} \mathrm{~N} / 116.143$ \\
\hline 22 & Dimethyl-ammonium & {$[\mathrm{DMNH}]^{+}$} & $\mathrm{C}_{2} \mathrm{H}_{8} \mathrm{~N} / 46.091$ \\
\hline 23 & $\begin{array}{l}\text { 2-Hydroxyethyl- } \\
\text { trimethylammonium }\end{array}$ & {$[\mathrm{HeTNH}]^{+}$} & $\mathrm{C}_{5} \mathrm{H}_{14} \mathrm{NO} / 104.17$ \\
\hline 24 & Methyl-ammonium & {$[\mathrm{MNH}]^{+}$} & $\mathrm{CH}_{6} \mathrm{~N} / 32.064$ \\
\hline 25 & Ethyl-ammonium & {$[\mathrm{ENH}]^{+}$} & $\mathrm{C}_{2} \mathrm{H}_{8} \mathrm{~N} / 46.091$ \\
\hline 26 & Propyll-ammonium & {$[\mathrm{PNH}]^{+}$} & $\mathrm{C}_{3} \mathrm{H}_{10} \mathrm{~N} / 60.118$ \\
\hline 27 & $\begin{array}{l}\text { Hexyldiethylmethylphosp } \\
\text { honium }\end{array}$ & {$[\mathrm{HDEMP}]^{+}$} & $\mathrm{C}_{11} \mathrm{H}_{26} \mathrm{P} / 189.3$ \\
\hline 28 & $\begin{array}{l}\text { Hexyltrimethylphosphoni } \\
\text { um }\end{array}$ & {$[\mathrm{HTMP}]^{+}$} & $\mathrm{C}_{9} \mathrm{H}_{22} \mathrm{P} / 161.24$ \\
\hline 29 & $\begin{array}{l}\text { Octyltrimethylphosphoniu } \\
\text { m }\end{array}$ & {$[\mathrm{OTMP}]^{+}$} & $\mathrm{C}_{12} \mathrm{H}_{22} \mathrm{P} / 204.33$ \\
\hline 30 & $\begin{array}{l}\text { Octyldiethylmethylphosp } \\
\text { honium }\end{array}$ & {$[\mathrm{ODEMP}]^{+}$} & $\mathrm{C}_{13} \mathrm{H}_{30} \mathrm{P} / 217.35$ \\
\hline 31 & $\begin{array}{l}\text { Diethyldimethylpropyl } \\
\text { phosphonium }\end{array}$ & $\underset{+}{[\mathrm{DEDMPP}]}$ & $\mathrm{C}_{9} \mathrm{H}_{22} \mathrm{P} / 161.24$ \\
\hline 32 & $\begin{array}{l}\text { Tributylmethylphosphoni } \\
\text { um }\end{array}$ & {$[\mathrm{TBMP}]^{+}$} & $\mathrm{C}_{13} \mathrm{H}_{30} \mathrm{P} / 217.35$ \\
\hline 33 & Tetrabutylphosphonium & {$[\mathrm{TBP}]^{+}$} & $\mathrm{C}_{16} \mathrm{H}_{36} \mathrm{P} / 259.43$ \\
\hline 34 & $\begin{array}{l}\text { Triisobutylmethylphosph } \\
\text { onium }\end{array}$ & {$\left[\mathrm{TIBMP}^{+}\right.$} & $\mathrm{C}_{13} \mathrm{H}_{30} \mathrm{P} / 217.35$ \\
\hline
\end{tabular}

\subsection{Selectivity of Pyrrolidinium Based Ionic Liquids}

Selectivity of pyrrolidinium based ionic liquids, $[\mathrm{OMPYR}]\left[\mathrm{BF}_{4}\right], \quad[\mathrm{HMPYR}]\left[\mathrm{BF}_{4}\right], \quad[\mathrm{BEPYR}]\left[\mathrm{CF}_{3} \mathrm{SO}_{3}\right]$, [DPPYR] $\left[\mathrm{CF}_{3} \mathrm{SO}_{3}\right]$ and [OMPYR] $\left[\mathrm{CF}_{3} \mathrm{SO}_{3}\right]$ are 13265 , $12215,11231,11072$ and 7761 respectively as shown in Fig. 4. They are similar as obtained for phosphonium based ionic liquids. As observed in previous case (phosphonium), anions $\left[\mathrm{CF}_{3} \mathrm{SO}_{3}\right]^{-}, \quad\left[\mathrm{BF}_{4}\right]^{-}$and $\quad[\mathrm{BMA}]^{-}$possess higher selectivity and are common for both phosphonium and pyrrolidinium based ionic liquids. 


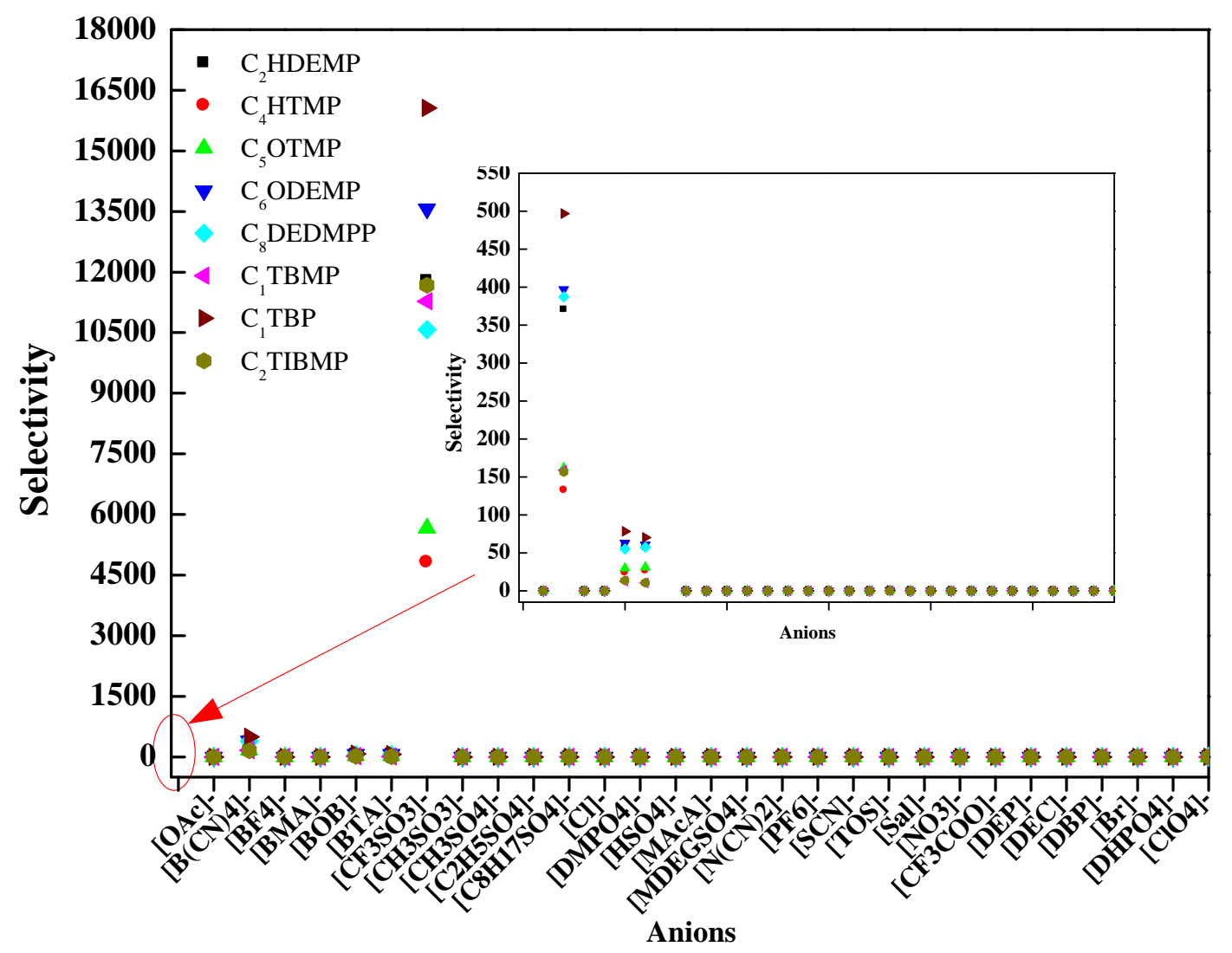

Figure 3. Plot of selectivity at infinite dilution $(T=298.15 \mathrm{~K})$ for phosphonium based ionic liquids with phthalic acid.

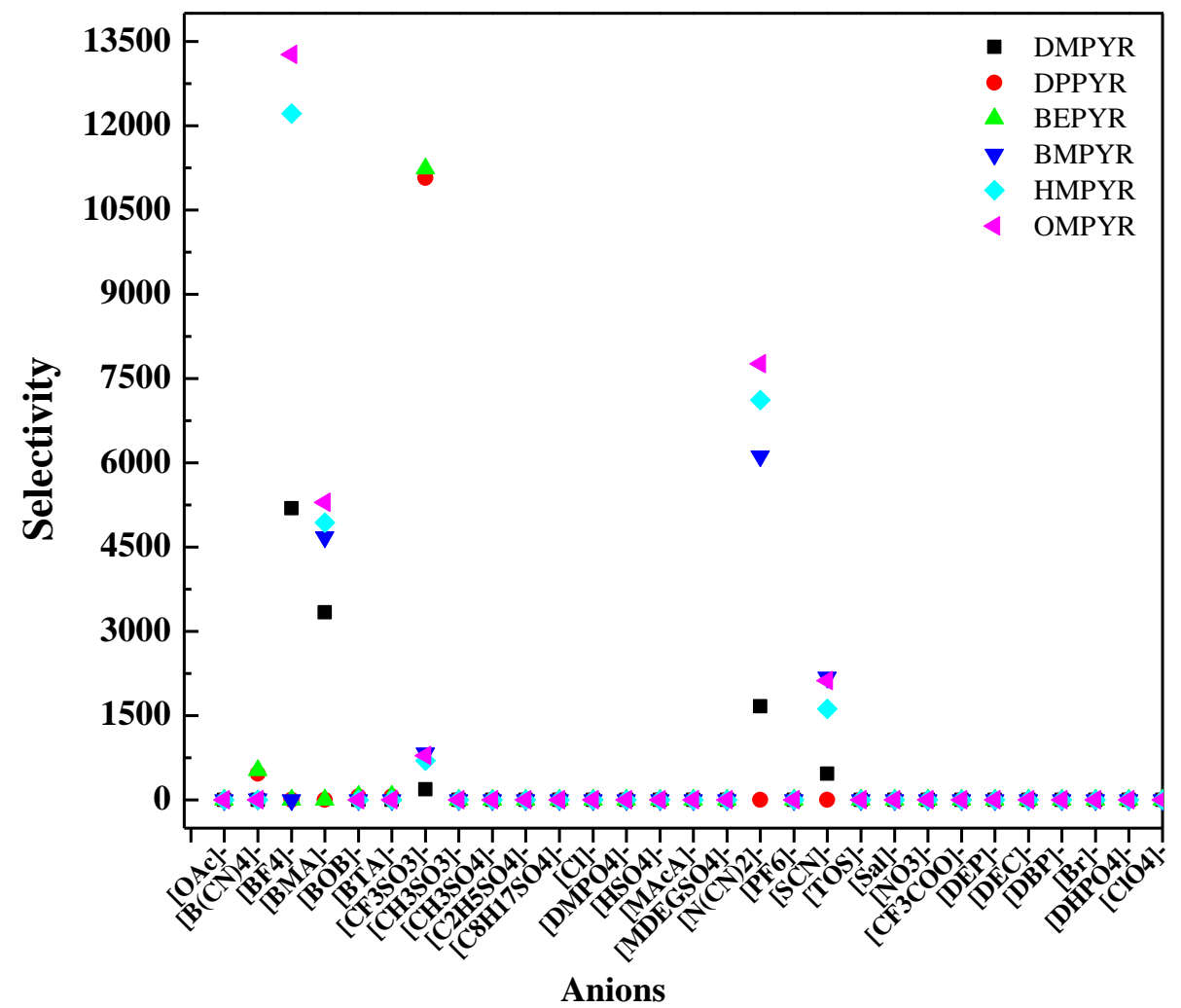

Figure 4. Plot of selectivity at infinite dilution $(T=298.15 \mathrm{~K})$ for pyrrolidinium based ionic liquids with phthalic acid. 
Table 4. Selected potential ionic liquids containing phosphonium and pyrrolidinium cations at T=298.15 K for phthalic acid.

\begin{tabular}{|c|c|c|c|c|c|c|c|}
\hline \multicolumn{4}{|c|}{ Phosphonium based ILs } & \multicolumn{4}{|c|}{ Pyrrolidinium bases ILs } \\
\hline $\begin{array}{l}\text { S. } \\
\text { No. }\end{array}$ & Name of the selective IL & Selectivity & $\gamma_{\text {water }}^{\infty}$ & $\begin{array}{c}\text { S. } \\
\text { No. }\end{array}$ & Name of the selective IL & Selectivity & $\gamma_{\text {water }}^{\infty}$ \\
\hline 1 & {$[\mathrm{TBP}]\left[\mathrm{CF}_{3} \mathrm{SO}_{3}\right]$} & 16057 & 1.605 & 1 & {$[\mathrm{OMPYR}]\left[\mathrm{BF}_{4}\right]$} & 13265 & 1.326 \\
\hline 2 & {$[\mathrm{ODEMP}]\left[\mathrm{CF}_{3} \mathrm{SO}_{3}\right]$} & 13565 & 1.356 & 2 & {$[\mathrm{HMPYR}]\left[\mathrm{BF}_{4}\right]$} & 12215 & 1.221 \\
\hline 3 & {$\left[\mathrm{HDFMP}^{2}\left[\mathrm{CF}_{2} \mathrm{SO}_{2}\right]\right.$} & 11783 & 1178 & 3 & {$[\mathrm{BEPYR}]\left[\mathrm{CF}_{3} \mathrm{SO}_{3}\right]$} & 11231 & 1.123 \\
\hline 3 & {$[\mathrm{HDEMP}]\left[\mathrm{CF}_{3} \mathrm{SO}_{3}\right]$} & $11 / 83$ & 1.178 & 4 & {$[\mathrm{DPPYR}]\left[\mathrm{CF}_{3} \mathrm{SO}_{3}\right]$} & 11072 & 1.107 \\
\hline 4 & {$[\mathrm{TIBMP}]\left[\mathrm{CF}_{3} \mathrm{SO}_{3}\right]$} & 11670 & 1.167 & 5 & {$[\mathrm{OMPYR}]\left[\mathrm{CF}_{3} \mathrm{SO}_{3}\right]$} & 7761 & 0.776 \\
\hline 5 & {$[\mathrm{TBMP}]\left[\mathrm{CF}_{3} \mathrm{SO}_{3}\right]$} & 11273 & 1.127 & 6 & {$[\mathrm{HMPYR}]\left[\mathrm{CF}_{3} \mathrm{SO}_{3}\right]$} & 7118 & 0.711 \\
\hline 6 & {$[\mathrm{DEDMPP}]\left[\mathrm{CF}_{3} \mathrm{SO}_{3}\right]$} & 10571 & 1.057 & 7 & {$[\mathrm{BMPYR}]\left[\mathrm{CF}_{3} \mathrm{SO}_{3}\right]$} & 6123 & 0.612 \\
\hline 7 & {$[\mathrm{OTMP}]\left[\mathrm{CF}_{3} \mathrm{SO}_{3}\right]$} & 5660 & 1.132 & 8 & [OMPYR][BMA] & 5297 & 0.529 \\
\hline 8 & {$[\mathrm{HTMP}]\left[\mathrm{CF}_{3} \mathrm{SO}_{3}\right]$} & 4808 & 0.961 & 9 & DMPYR $\left[\mathrm{BE}_{4}\right]$ & 5193 & 1308 \\
\hline 9 & {$[\mathrm{TBP}]\left[\mathrm{BCN}_{4}\right]$} & 496 & 18.483 & 9 & DIVIPYK $]\left[\mathrm{BF}_{4}\right]$ & 5193 & 1.508 \\
\hline
\end{tabular}

Table 5. Selected potential ionic liquids containing imidazolium and ammonium cations at T=298.15 for phthalic acid.

\begin{tabular}{|c|c|c|c|c|c|c|c|}
\hline \multicolumn{4}{|c|}{ Imidazolium based ILs } & \multicolumn{4}{|c|}{ Ammonium based ILs } \\
\hline $\begin{array}{l}\text { S. } \\
\text { No. }\end{array}$ & Name of the selective IL & Selectivity & $\gamma_{\text {water }}^{\infty}$ & S. No. & Name of the selective IL & Selectivity & $\gamma_{\text {water }}^{\infty}$ \\
\hline 1 & {$\left[\mathrm{C}_{4} \mathrm{DMIM}\right]\left[\mathrm{CF}_{3} \mathrm{SO}_{3}\right]$} & 11678 & 1.167 & 1 & {$[\mathrm{MNH}][\mathrm{DEC}]$} & 9650 & 1.930 \\
\hline 2 & {$\left[\mathrm{C}_{8} \mathrm{MIM}\right][\mathrm{SCN}]$} & 10013 & 1.001 & 2 & [PNH][DBP] & 8228 & 0.822 \\
\hline 3 & {$\left[\mathrm{C}_{2} \mathrm{DMIM}\right]\left[\mathrm{CF}_{3} \mathrm{SO}_{3}\right]$} & 10155 & 1.015 & 3 & {$[\mathrm{ENH}][\mathrm{DBP}]$} & 7953 & 0.795 \\
\hline 4 & {$\left[\mathrm{C}_{6} \mathrm{MIM}\right][\mathrm{SCN}]$} & 8737 & 0.873 & 4 & {$[\mathrm{HENTNH}]\left[\mathrm{BF}_{4}\right]$} & 7660 & 0.766 \\
\hline 5 & {$\left[\mathrm{C}_{5} \mathrm{MIM}\right][\mathrm{SCN}]$} & 8196 & 0.819 & 5 & {$[\mathrm{BTNH}]\left[\mathrm{CF}_{3} \mathrm{SO}_{3}\right]$} & 3863 & 0.772 \\
\hline 6 & {$\left[\mathrm{C}_{4} \mathrm{MIM}\right][\mathrm{SCN}]$} & 7546 & 0.754 & 6 & [PNH][DEP] & 3435 & 0.687 \\
\hline 7 & {$\left[\mathrm{C}_{2} \mathrm{MIM}\right][\mathrm{SCN}]$} & 6515 & 0.651 & 7 & {$[\mathrm{HETNH}]\left[\mathrm{CF}_{3} \mathrm{SO}_{3}\right]$} & 3370 & 0.337 \\
\hline 8 & {$\left[\mathrm{C}_{1} \mathrm{MIM}\right][\mathrm{SCN}]$} & 6216 & 0.621 & 8 & [HETNH][BMA] & 3291 & 0.329 \\
\hline 9 & {$\left[\mathrm{C}_{1} \mathrm{DMIM}\right]\left[\mathrm{MDGSO}_{4}\right]$} & 2893 & 0.289 & 9 & {$[\mathrm{ENH}][\mathrm{OAC}]$} & 3311 & 0.331 \\
\hline 10 & {$\left[\mathrm{C}_{1} \mathrm{DMIM}\right][\mathrm{BMA}]$} & 2844 & 0.284 & 10 & {$[\mathrm{BETNH}]\left[\mathrm{CF}_{3} \mathrm{SO}_{3}\right]$} & 2961 & 0.888 \\
\hline $\begin{array}{l}11 \\
12\end{array}$ & $\begin{array}{l}{\left[\mathrm{C}_{8} \mathrm{MIM}\right]\left[\mathrm{CF}_{3} \mathrm{SO}_{3}\right]} \\
{\left[\mathrm{C}_{4} \mathrm{DMIM}\right]\left[\mathrm{BCN}_{4}\right]}\end{array}$ & $\begin{array}{c}2598 \\
639\end{array}$ & $\begin{array}{l}1.199 \\
17.52\end{array}$ & 11 & {$[\mathrm{ENH}][\mathrm{DEP}]$} & 2192 & 0.657 \\
\hline 13 & {$\left[\mathrm{C}_{2} \mathrm{DMIM}\right]\left[\mathrm{BCN}_{4}\right]$} & 518 & 14.88 & & & & \\
\hline
\end{tabular}

The selective ionic liquids and the corresponding aqueous phase activity coefficient of phthalic acid with phosphonium and pyrrolidinium based ionic liquids at infinite dilution $(T=298.15 \mathrm{~K})$ are shown in Table 4 . Cations [OMPYR], [HMPYR], [BEPYR], [DPPYR], DMPYR] and [BMPYR] are not suitable extractants with most of the anions (except for $\left[\mathrm{CF}_{3} \mathrm{SO}_{3}\right]^{-},\left[\mathrm{BF}_{4}\right]^{-},[\mathrm{SCN}]^{-}$ and $\left[\mathrm{BMA}^{-}\right)$having very low selectivity values. From Fig. 4 it can be seen that apart from $\left[\mathrm{CF}_{3} \mathrm{SO}_{3}\right]^{-},\left[\mathrm{BF}_{4}\right]^{-},[\mathrm{SCN}]^{-}$ and $[\mathrm{BMA}]^{-}$, selectivity values for ionic liquids with these anions were very small, less than unity in most of the cases, and some near to zero. Thus the cations [OMPYR], [HMPYR], [BEPYR], [DPPYR], [DMPYR] and [BMPYR] are undesirable for the extraction of phthalic acid esters from aqueous phase.

\subsection{Selectivity of Imidazolium Based Ionic Liquids}

The selectivity values of imidazolium based ionic liquids were shown in Fig. 5. High selectivity values were obtained by $\left[\mathrm{C}_{4} \mathrm{DMIM}\right]\left[\mathrm{CF}_{3} \mathrm{SO}_{3}\right], \quad\left[\mathrm{C}_{8} \mathrm{MIM}\right][\mathrm{SCN}]$, $\left[\mathrm{C}_{2} \mathrm{DMIM}\right]\left[\mathrm{CF}_{3} \mathrm{SO}_{3}\right],\left[\mathrm{C}_{6} \mathrm{MIM}\right][\mathrm{SCN}],\left[\mathrm{C}_{5} \mathrm{MIM}\right][\mathrm{SCN}]$ and $\left[\mathrm{C}_{4} \mathrm{MIM}\right][\mathrm{SCN}]$. The corresponding selectivity values were $11678,10013,10155,8737,8196$ and 7546 respectively for the extraction of phthalic acid. Here the favorable anions were $\left[\mathrm{CF}_{3} \mathrm{SO}_{3}\right]^{-}$, $[\mathrm{SCN}]^{-}$, [BMA] $]^{-}$, and $\left[\mathrm{B}(\mathrm{CN})_{4}\right]^{-}$. From Fig. 5 , it can be observed that the selective cations follow the order: $\left[\mathrm{C}_{4} \mathrm{DMIM}\right]^{+}>\left[\mathrm{C}_{8} \mathrm{MIM}\right]^{+}>\left[\mathrm{C}_{2} \mathrm{DMIM}\right]^{+}>\left[\mathrm{C}_{6} \mathrm{MIM}\right]^{+}$ $>\left[\mathrm{C}_{5} \mathrm{MIM}\right]^{+}>\left[\mathrm{C}_{4} \mathrm{MIM}\right]^{+}$. Anions $\left[\mathrm{B}(\mathrm{CN})_{4}\right]^{-},[\mathrm{BOB}]^{-}$, $[\mathrm{BTA}]^{-},\left[\mathrm{CF}_{3} \mathrm{SO}_{3}\right]^{-},[\mathrm{SCN}]^{-}$and $\left[\mathrm{PF}_{6}\right]^{-}$gave high selectivity values. It should be noted that $\left[\mathrm{C}_{1} \mathrm{DMIM}\right]^{+}$having interaction with anions: $\left[\mathrm{BMA}^{-}, \quad[\mathrm{BOB}]^{-}, \mathrm{BBT}^{-}\right.$, $\left[\mathrm{CF}_{3} \mathrm{SO}_{3}\right]^{-},\left[\mathrm{HSO}_{4}\right]^{-},\left[\mathrm{MDEGSO}_{4}\right]^{-},\left[\mathrm{N}(\mathrm{CN})_{2}\right]^{-},\left[\mathrm{PF}_{6}\right]^{-}$, and $[\mathrm{SCN}]^{-}$gave higher values of selectivities when compared with other cations. The best selectivity values and the aqueous phase activity coefficient of phthalic acid with imidazolium based ionic liquids at infinite dilution $(T=$ $298.15 \mathrm{~K}$ ) are shown in Table 5.

\subsection{Selectivity of Pyridinium and Ammonium Based Ionic Liquids}

Fig. 6 shows the selectivity of the pyridinium based ILs with phthalic acid. Within the high values of selectivity were reported such as: $[\mathrm{OMPYR}]\left[\mathrm{BF}_{4}\right]$ (13265) > $[\mathrm{HMPYR}]\left[\mathrm{BF}_{4}\right](12215)>[\mathrm{BEPYR}]\left[\mathrm{CF}_{3} \mathrm{SO}_{3}\right](11231)>$ $[\mathrm{DPPYR}]\left[\mathrm{CF}_{3} \mathrm{SO}_{3}\right](11072)>$ [OMPYR $]-\left[\mathrm{CF}_{3} \mathrm{SO}_{3}\right](7761)$ $>$ [HMPYR $]\left[\mathrm{CF}_{3} \mathrm{SO}_{3}\right]$ (7118). In case of ammonium based cations as shown in Fig. 7, the selectivity pattern followed: $[\mathrm{MNH}][\mathrm{DEC}](9650)>[\mathrm{PNH}][\mathrm{DBP}]$ (8228) > $[\mathrm{ENH}][\mathrm{DBP}](7953)>[\mathrm{HEN}-\mathrm{TNH}]\left[\mathrm{BF}_{4}\right]$ (7660) > $[\mathrm{BTNH}]\left[\mathrm{CF}_{3} \mathrm{SO}_{3}\right]$ (3863). It can be seen that for phthalic acid, the selectivity increases with an increase in the alkyl chain length on most of the cations. This was due to the increasing van der Waals volume which leads to increasing entrapment of the phthalic acid in the cation structure. Among all five groups of cations the selectivity order followed: phosphonium $>$ pyrrolidinium $>$ imidazolium $>$ ammonium $>$ pyridinium. Table 5 and 6 represents the selective values and the aqueous phase activity coefficient of phthalic acid with ammonium and pyridinium based ionic liquids at infinite dilution $(\mathrm{T}=298.15 \mathrm{~K})$. Higher values of selectivity as well as high values $(>1)$ of $\gamma_{1}^{\infty}$ water 
are essential for the selection of ionic liquid for extraction. The above results have guided us in future to prepare the supported ionic liquid membrane (SILM) or bulk ionic liquid membrane (BILM) for liquid liquid extraction based on ionic liquids.

\section{Solvent Capacity}

The solvent capacity at infinite dilution was predicted by COSMO-RS model. Solvent capacity data was obtained from equation (2). Figure 8 shows the solvent capacity of imidazolium based cations for phthalic acid. The maximum extraction capacity of 10,000 was observed for the imidazolium based cations. Cations $\left[\mathrm{C}_{1} \mathrm{DMIM}\right]^{+}$, $\left[\mathrm{C}_{2} \mathrm{MIM}\right]^{+}, \quad\left[\mathrm{C}_{4} \mathrm{DMIM}\right]^{+}, \quad\left[\mathrm{C}_{5} \mathrm{MIM}\right]^{+}, \quad\left[\mathrm{C}_{6} \mathrm{MIM}\right]^{+}$and $\left[\mathrm{C}_{8} \mathrm{MIM}\right]^{+}$, gave maximum capacity of 10,000 by interacting with anion $[\mathrm{SCN}]^{-}$. Similarly the cations $\left[\mathrm{C}_{1} \mathrm{DMIM}\right]^{+}$with $\left[\mathrm{MDEGSO}_{4}\right]^{-},\left[\mathrm{C}_{1} \mathrm{DMIM}\right]^{+}$with $\left[\mathrm{HSO}_{4}\right]^{-}$, $\left[\mathrm{C}_{2} \mathrm{DMIM}\right]^{+}$with $\left[\mathrm{CF}_{3} \mathrm{SO}_{3}\right]^{-}$and $\left[\mathrm{C}_{4} \mathrm{DMIM}\right]^{+}$with $\left[\mathrm{CF}_{3} \mathrm{SO}_{3}\right]^{-}$ also gave solvent capacity of 10,000. Solvent capacity for pyridinium based cations was only $\sim 3333$ for $\left[\mathrm{C}_{2} \mathrm{MPY}\right]^{+}$, $\left[\mathrm{C}_{4} \mathrm{MPY}\right]^{+},\left[\mathrm{C}_{6} \mathrm{MPY}\right]^{+}$and $\left[\mathrm{C}_{8} \mathrm{MPY}\right]^{+}$with anion $\left[\mathrm{CF}_{3} \mathrm{SO}_{3}\right]^{-}$. All anions except $\left[\mathrm{CF}_{3} \mathrm{SO}_{3}\right]^{-}$with $\left[\mathrm{C}_{2} \mathrm{MPY}\right]^{+},\left[\mathrm{C}_{4} \mathrm{MPY}\right]^{+}$, $\left[\mathrm{C}_{6} \mathrm{MPY}\right]^{+}$and $\left[\mathrm{C}_{8} \mathrm{MPY}\right]^{+}$possessed unfa-vorable solvent capacity. In case of pyrrolidinium based cations, the maximum capacity of solvent was 10,000 for $\left([\mathrm{HMPYR}]^{+}\right.$ and $[\mathrm{OMPYR}]^{+}$with $\left[\mathrm{BF}_{4}\right]^{-},[\mathrm{DPPYR}]^{+}$and $[\mathrm{BEPYR}]^{+}$with $\left[\mathrm{CF}_{3} \mathrm{SO}_{3}\right]^{-}$, [BMPYR $]^{+}$, [HMPYR $]^{+}$and $[\mathrm{OMPYR}]^{+}$with $\left[\mathrm{HSO}_{4}\right]^{-}$and anions $\left[\mathrm{MDEGSO}_{4}\right]^{-}$and $\left[\mathrm{N}(\mathrm{CN})_{2}\right]^{-}$with cations $[\mathrm{BMPYR}]^{+}, \mathrm{HHMPY}^{+}$and $[\mathrm{OMPYR}]^{+}$. All pyridinium based cations except $[\mathrm{DMPYR}]^{+}$and $[\mathrm{DPPYR}]^{+}$ gave selectivity of 10,000 with anion [BMA] (Figure not given). The highest capacity value obtained was 10,000 .
Ionic Liquids: $[\mathrm{DMNH}][\mathrm{TOS}]^{-}, \quad[\mathrm{HETNH}]\left[\mathrm{BF}_{4}\right]-$ and [HETNH]-[MDEGSO $]^{-}$gave maximum selectivity of 10,000. In case of phosphonium based cation (Figure not shown) the maximum solvent capacity was obtained by the anion $\left[\mathrm{CF}_{3} \mathrm{SO}_{3}\right]^{-}$. Favorable cations are $[\mathrm{HDEMP}]^{+}$, $\left.[\mathrm{ODEMP}]^{+},{ }_{\mathrm{TBMP}}\right]^{+},[\mathrm{TBP}]^{+}$and $[\mathrm{TIBMP}]^{+}$. All cations except $[\mathrm{HTMP}]^{+}$and $[\mathrm{OTMP}]^{+}$are unfavorable as the activity coefficient of phthalic in ionic liquid phase is zero. It should be noted that the selectivity and capacity are functions of infinite dilution activity coefficients. In some of the above Figures (3-8) the symbols are overlapping each other which confirm that different ionic liquids having the same selectivity or capacity value.

Table 6. Selected potential ionic liquids containing pyridinium cations at $T=298.15$ for phthalic acid.

\begin{tabular}{clcc}
\hline \multicolumn{4}{c}{ Pyridinium based ILs } \\
S. No. & $\begin{array}{c}\text { Name of the selective } \\
\text { IL }\end{array}$ & $\begin{array}{c}\text { Selectiv } \\
\text { ity }\end{array}$ & $\gamma_{\text {water }}^{\infty}$ \\
\hline 1 & {$\left[\mathrm{C}_{8} \mathrm{MPY}\right]\left[\mathrm{CF}_{3} \mathrm{SO}_{3}\right]$} & 4735 & 1.42 \\
2 & {$\left[\mathrm{C}_{6} \mathrm{MPY}\right]\left[\mathrm{CF}_{3} \mathrm{SO}_{3}\right]$} & 4205 & 1.261 \\
3 & {$\left[\mathrm{C}_{4} \mathrm{MPY}\right]\left[\mathrm{CF}_{3} \mathrm{SO}_{3}\right]$} & 3582 & 1.07 \\
4 & {$\left[\mathrm{C}_{2} \mathrm{MPY}\right]\left[\mathrm{CF}_{3} \mathrm{SO}_{3}\right]$} & 3150 & 0.945 \\
5 & {$\left[\mathrm{C}_{8} \mathrm{MPY}\right]\left[\mathrm{BCN}_{4}\right]$} & 271 & 13.99 \\
6 & {$\left[\mathrm{C}_{6} \mathrm{MPY}\right]\left[\mathrm{BCN}_{4}\right]$} & 257 & 12.162 \\
7 & {$\left[\mathrm{C}_{4} \mathrm{MPY}\right]\left[\mathrm{BCN}_{4}\right]$} & 180 & 8.77 \\
8 & {$\left[\mathrm{C}_{2} \mathrm{MPY}\right]\left[\mathrm{BCN}_{4}\right]$} & 154.9 & 7.855 \\
\hline
\end{tabular}

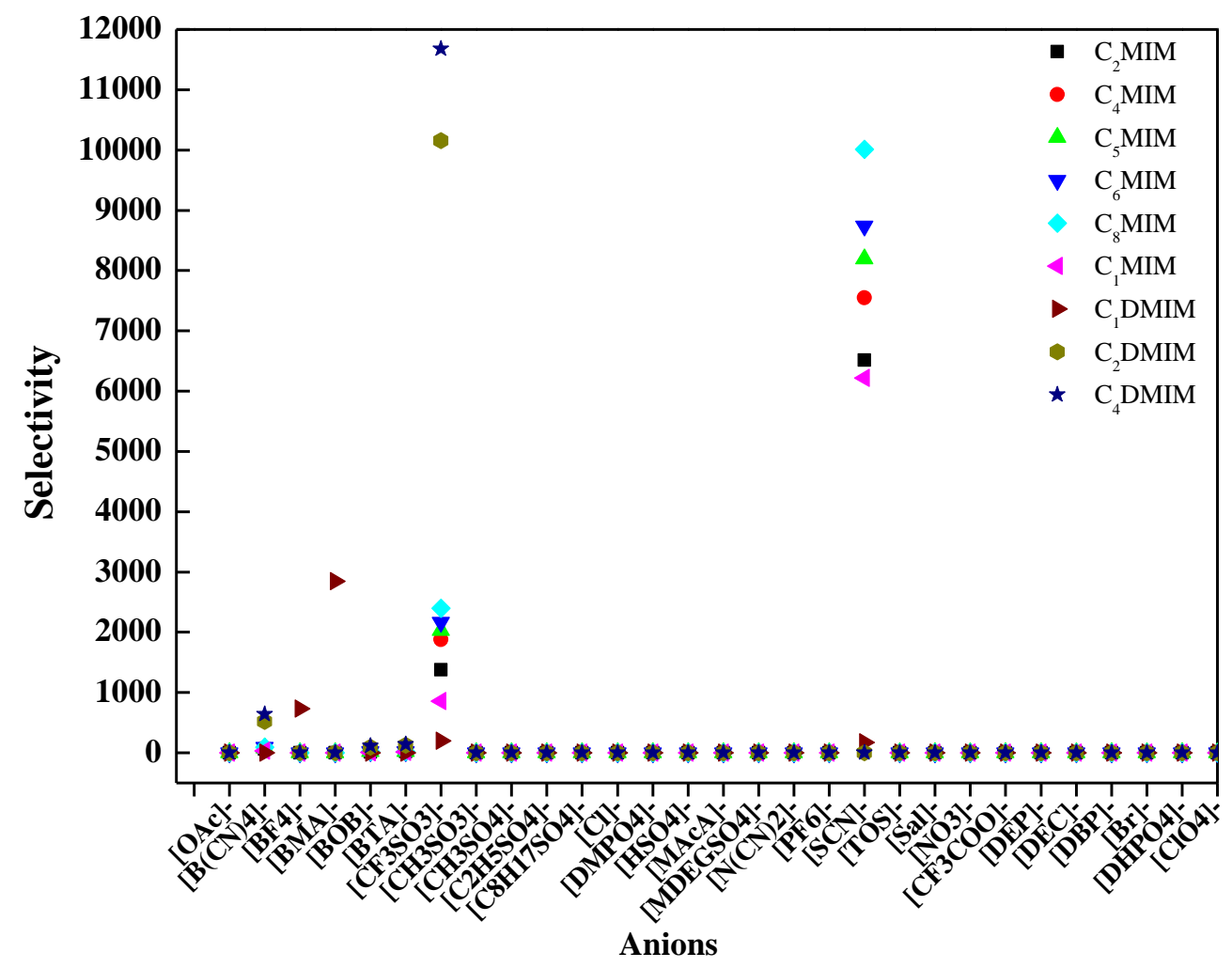

Figure 5. Selectivity at infinite dilution and at room temperature (T=298.15 K) for imidazolium based ionic liquids. 


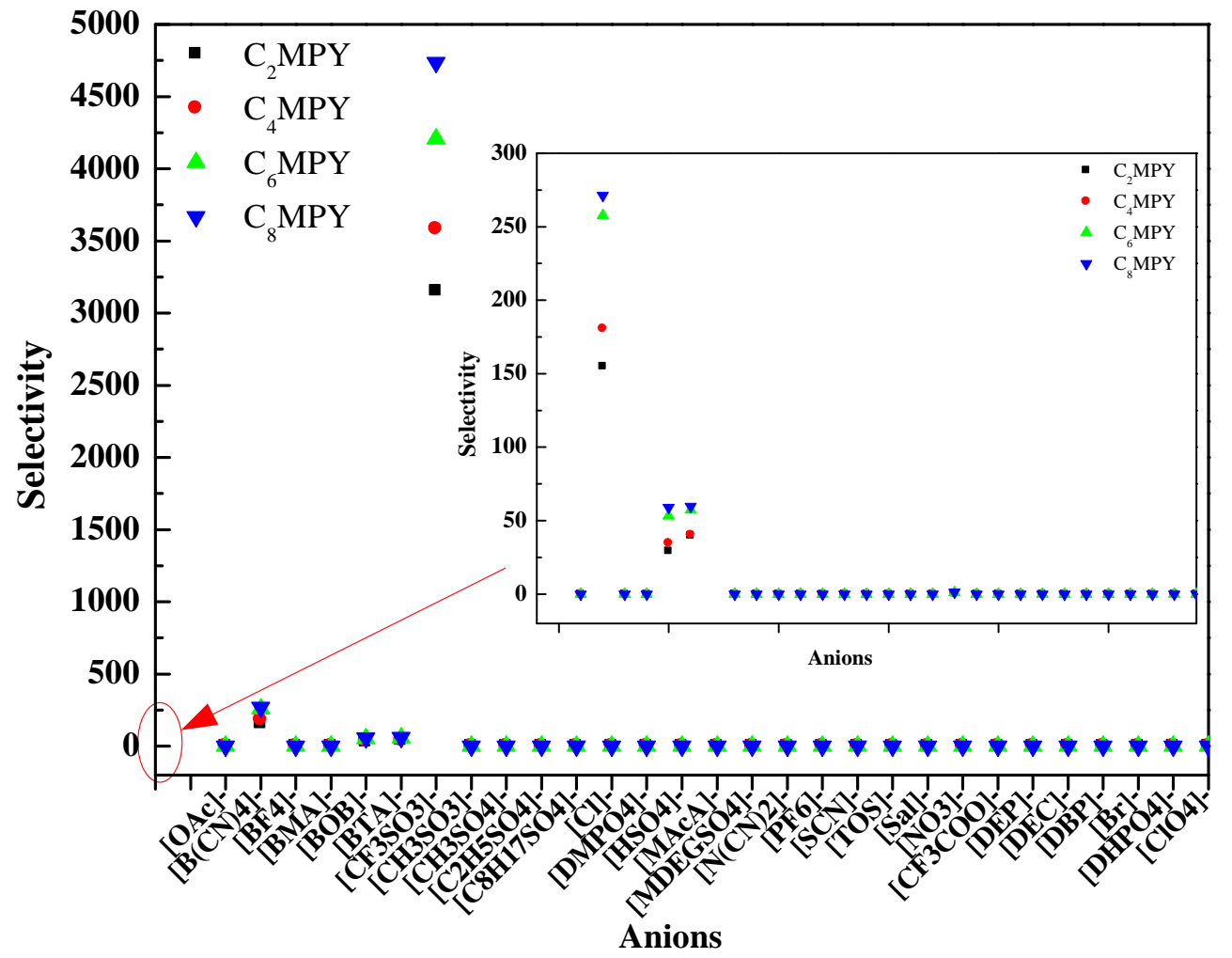

Figure 6. Selectivity at infinite dilution and at room temperature $(T=298.15 \mathrm{~K})$ for pyridinium based ionic liquids with phthalic acid.

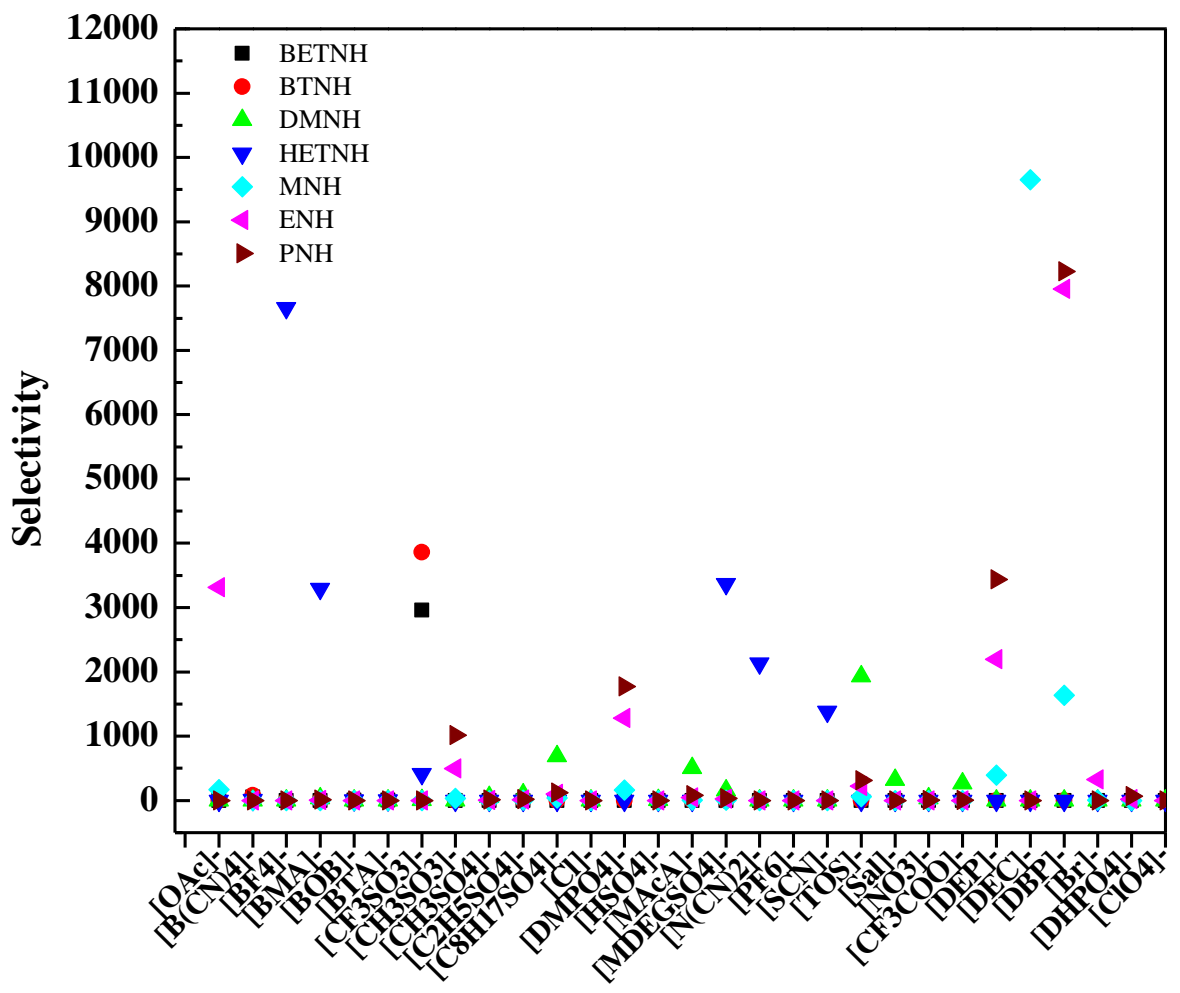

Anions

Figure 7. Selectivity at infinite dilution and at room temperature $(T=298 \mathrm{~K})$ for ammonium based ionic liquids with phthalic acid. 


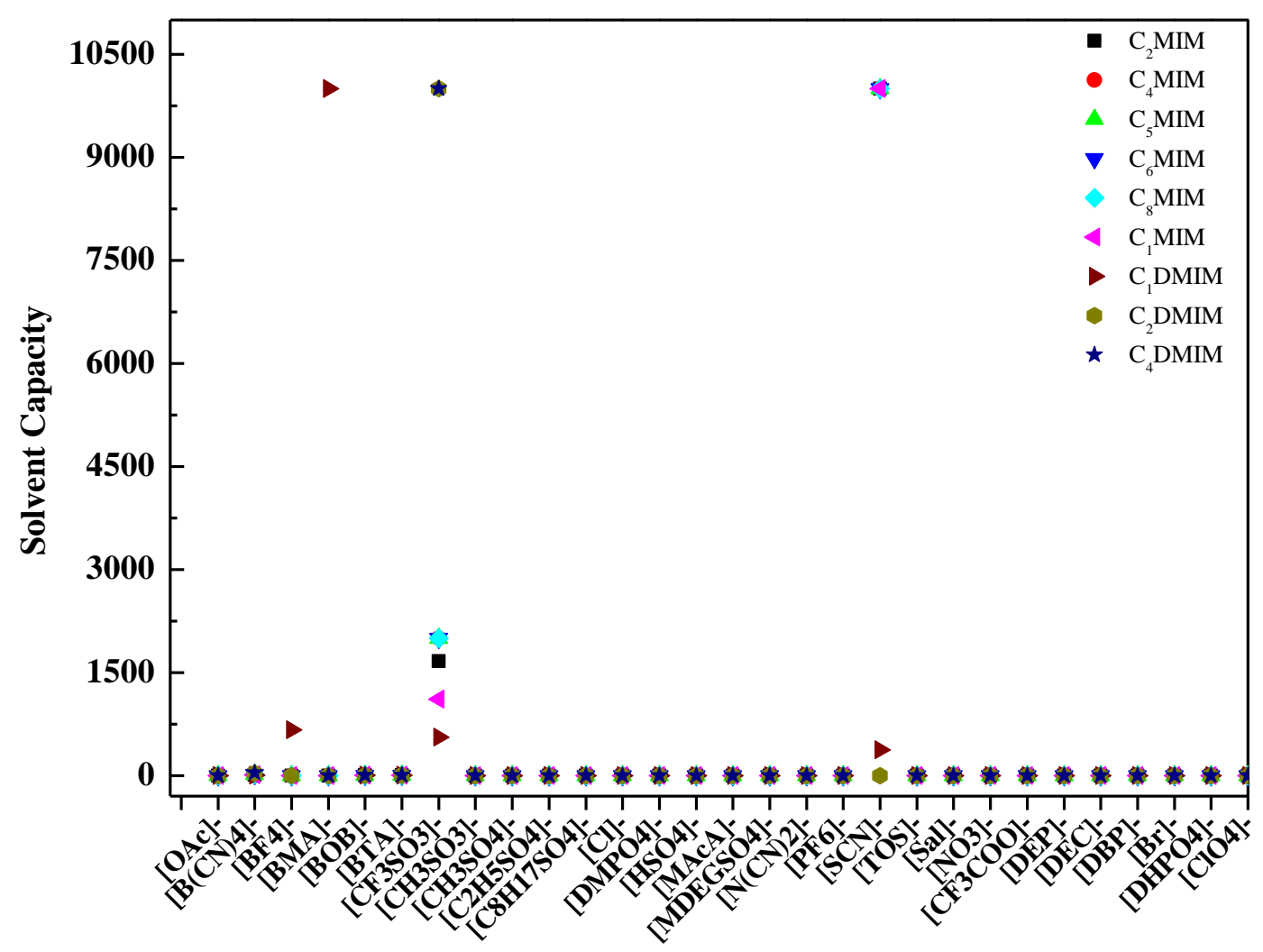

Anions

Figure 8. Solvent capacity at infinite dilution and at ambient temperature $(T=298.15 \mathrm{~K})$ for imidazolium based cations with phthalic acid.

\section{Conclusions}

This study reports the screening of 34 cations and 29 anions i.e. 986 possible ionic liquids to determine the best extractant for the extraction of phthalic acid from aqueous phase. Initially benchmarking was done by comparing experimental logarithmic octanol-water partition coefficient values $\left(\log \left(K_{\text {ow }}\right)\right)$ with COSMO-RS predicted data on selected phthalic acid esters (PE) which were similar in structure to that of phthalic acid. Thereafter, different groups of cations such as phosphonium, imidazolium, pyridinium, pyrrolidinium and ammonium were investigated. All the selectivity calculations were performed using infinite dilution activity coefficients as an indicator for the screening of potential ionic liquids. Among the screened cations the best selectivity order follows: phosphonium $>$ pyrolidinium $>$ imidazolium $>$ ammonim $>$ pyridinium. Corresponding selectivity values with the best anion were $[\mathrm{TBP}]\left[\mathrm{CF}_{3} \mathrm{SO}_{3}\right](16057)>[\mathrm{OMPYR}]\left[\mathrm{BF}_{4}\right]$ $(13265)>\left[\mathrm{C}_{4} \mathrm{DMIM}\right]\left[\mathrm{CF}_{3} \mathrm{SO}_{3}\right](11678)>[\mathrm{MNH}][\mathrm{DEC}]$ $(9650)>\left[\mathrm{C}_{8} \mathrm{MPY}\right]\left[\mathrm{CF}_{3} \mathrm{SO}_{3}\right]$ (4735). The results of this study are encouraging which can make the ionic liquids as a potential solvent for the treatment of water and wastewater containing organic effluents like phthalic acid.

\section{Acknowledgments}

Authors gratefully acknowledge that this work was financially supported by the research grant under the Fast Track Scheme from the Department of Science and Technology (DST), Government of India vide sanction No. SR/FTP/ETA-56/2010.

\section{References}

[1] R. Rudel, L. Perovich Endocrine disrupting chemicals in indoor and outdoor air, Atmos. Environ., 4317, 170-181, 2009.

[2] J. Rudolph, J. R. Robert, Plasticizers from Plastic Devices: Extraction Metabolism and Accumulation by Biological Systems, Science, 170, 460-462, 1970.

[3] E. Carlsen, A. Giwercman, N. Keiding, N. E. Skakkebaek, Evidence for decreasing quality of semen during past 50 years, Br. Med. J., 305, 609-613, 1992.

[4] E. Diamanti-Kandarakis, J. Bourguignon, L. C. Giudice, R. Hauser, G. S. Prins, A. M. Soto, R. T. Zoeller, A. C. Gore, Endocrine-Disrupting Chemicals: An Endocrine Society Scientific Statement, Endocr. Rev., 304, 293-342, 2009.

[5] C. Sonnenschein, A. M. Soto, An updated review of environmental estrogen and androgen mimics and antagonists, J. Steroid Biochem. Mol. Biol., 65, 143-150, 1998.

[6] H. S. Chang, K. H. Choo, B. Lee, S. J. Choi, Review: The methods of identification analysis and removal of endocrine disrupting compounds EDCs in water, $J$. Hazard.Mater., 1721, 1-12, 2009.

[7] A. M. Comerton, R. C. Andrews, D. M. Bagley, P. Yang, Membrane adsorption of endocrine disrupting compounds and pharmaceutically active compounds, $J$. Membr. Sci., 303, 267-277, 2007. 
[8] S. A. Snyder et al., Role of membranes and activated carbon in the removal of endocrine disruptors and pharmaceuticals, Desalination 202, 156-181, 2007.

[9] B. Ning, N. Graham, Y. Zhang, M. Nakonechny, E. G. Mohamed, Degradation of Endocrine Disruptions Chemicals by Ozone/AOPs. Ozone: Science Engineering, J. Int. Ozone Assoc., 293, 153-176, 2007.

[10] T. C. Zhang, S. C. Emary, Jar tests for evaluation of Atrazine removal at drinking water treatment plants, Environ. Eng. Sci., 166, 417-432, 1999.

[11] T. A. Ternes, M. Meisenheimer, D. McDowell, F. Sacher, H. J. Brauch, B. H. Gulde, G. Preuss, U. Wilme, N. Z. Seibert, Removal of pharmaceuticals during drinking water treatment, Environ. Sci. Technol., 3617, 3855-3863, 2002.

[12] A. Gallegos-Muñoz, A. Zaleta-Aguilar, B. GonzálezRolón, On an Exergy Efficiency Definitionof a Wastewater Treatment Plant, Int. J. Thermodyn., 64, 169176,2003

[13] P. Wu, R.W. Field, R. England, B. J. Brisdon, A Fundamental Study of organo Function-alised PDMS Membranes for the Pervaporative Recovery of Phenolic Compounds from Aqueous Streams, J. Membr. Sci., 1902, 147-157, 2001.

[14] S. Mohanty, T. Banerjee, K. Mohanty, Quantum chemical based screening of ionic liquids for the extraction of phenol from aqueous solution, Ind. Eng. Chem. Res., 496, 2916-2925, 2010.

[15] D. J. Paulson, R. L. Wilson, D. D. Spatz, Cross flow membrane technology and its applications, Food Techno., 381, 77-87, 1984.

[16] S. D. Doig, A. T. Boam, A. G. Livingston, D. C. Stuckey, Mass transfer of hydrophobic solutes in solvent swollen silicone rubber membranes, J. Membr. Sci., 1541 127-140, 1999.

[17] Kislik, V. S. (2010). Liquid membranes principles and applications in chemical separations and wastewater treatment. 2010.

[18] B. Burghoff, E. L. V. Goetheer, A. B. de Haan, COSMO-RS-Based extractant screening for phenol extraction as model System, Ind. Eng. Chem. Res.,4712, 4263-4269, 2008.

[19] A. A. P. Kumar, T. Banerjee, Thiophene separation with ionic liquids for desulphur-ization: A quantum chemical approach, Fluid Phase Equilib., 278, 1-8, 2009.

[20] N. R. Varma, A. Ramalingam, T. Banerjee, Experiments correlations and COSMO-RS predictions for the extraction of benzothiophene from n-hexane using imidazolium-based ionic liquids, Chem. Eng. J., 1661, 30-39, 2011.

[21] K. Kedra-Krolik, M. Fabrice, J. Jaubert, Extraction of thiophene or pyridine from $n$-heptane using ionic liquids gasoline and diesel desulfurization, Ind. Eng. Chem. Res.,504, 2296-2306, 2011.

[22] L. Kumar, T. Banerjee, K. Mohanty, Prediction of selective extraction of cresols from aqueous solutions by ionic liquids using theoretical approach, Sep. Sci. Technol., 4613, 2075-2087, 2011.

[23] S. Potdar, R. Anantharaj, T. Banerjee, Aromatic extraction using mixed ionic liquids: experiments and COSMO-RS predictions, J. Chem. Eng. Data., 574, 1026-1035, 2012.

[24] S. R. Pilli, T. Banerjee, K. Mohanty, Extraction of pentachlorophenol and dichlorodiphenyltrichloroethane from aqueous solutions using ionic liquids, J. Ind. Eng. Chem., 186, 1983-1996, 2012.

[25] L. Y. Garcia-Chavez, A. J. Hermans, B. Schuur, A. B. de Haan, COSMO-RS assisted solvent screening for liquid-liquid extraction of mono ethylene glycol from aqueous streams, Sep. Purif. Technol., 97 ILSEPT 2011 special issue 2-10, 2012.

[26] R. Lü, J. Lin, Z. Qu, Theoretical study on interactions between ionic liquids and organosulfur compounds, Comp. Theor. Chem., 1002, 49-58, 2012.

[27] A. Klamt, G. Schuüürmann, COSMO: A New Approach to dielectric screening in solvents with explicit expressions for the screening energy and its gradient, $J$. Chem. Soc., Perkin Trans. 25, 799-805, 1993.

[28] S. Lin, S. I. Sandler, A priori phase equilibrium prediction from a segment contribution solvation model, Ind. Eng. Chem. Res., 415, 899-913, 2002.

[29] A. Klamt, COSMO-RS: From Quantum Chemistry to Fluid Phase Thermodynamics and Drug Design. 1st ed. 2005.

[30] T. Banerjee, A. Khanna, Infinite dilution activity coefficients for trihexyltetradecyl phosphonium ionic liquids: measurements and COSMO-RS prediction, Ind. Eng. Chem. Res., 516, 2170-2177, 2006A.

[31] T. Banerjee, M. K. Singh, A. Khanna, Prediction of binary VLE for imidazolium based ionic liquid systems using COSMO-RS, Ind. Eng. Chem. Res., 459, 32073219, 2006B.

[32] Frisch et al., GAUSSIAN 03 Revision C.01 Gaussian Inc. Pittsburgh PA, 2003.

[33] J. Fan, Y. Fan, Y. Pei, K. Wu, J. Wang, M. Fan, Solvent extraction of selected endocrine-disrupting phenols using ionic liquids, Sep. Purif. Technol., 613, 324-331, 2008.

[34] S. T. Lin, S. I. Sandler, Prediction of octanol-water partition coefficients using a group contribution solvation model, Ind. Eng. Chem. Res., 38, 4081-4091, 1999.

[35] R. Anantharaj, T. Banerjee, COSMO-RS-based screening of ionic liquids as green solvents in denitrification studies, Ind. Eng. Chem. Res., 4918, 8705$8725,2010$. 